DOI 10.35381/cm.v5i9.256

\title{
Harina integral de zapallo (cucúrbita moschata) para alimento alternativo en la producción avícola
}

\section{Wholemeal pumpkin flour (cucúrbita moschata) for alternative food in poultry production}

\author{
Freddy Alain Mendoza Rivadeneira \\ famendoza@utm.edu.ec \\ Universidad Técnica de Manabí, Portoviejo \\ Ecuador \\ https://orcid.org/0000-0003-1457-688X \\ Roy Leonardo Barre Zambrano \\ rolebaz@hotmail.com \\ Universidad Laica Eloy Alfaro de Manabí, Manta \\ Ecuador \\ https://orcid.org/0000-0002-4849-3532 \\ Plinio Abelardo Vargas Zambrano \\ pavargas@utm.edu.ec \\ Universidad Técnica de Manabí, Portoviejo \\ Ecuador \\ https://orcid.org/0000-0002-2152-7317 \\ Limberg Ivan Zambrano Pinoargote \\ lizambrano@utm.edu.ec \\ Universidad Técnica de Manabí, Portoviejo \\ Ecuador \\ https://orcid.org/0000-0003-4827-8637
}

Recibido: 1 de mayo de 2019

Aprobado: 10 de junio de 2019 


\title{
RESUMEN
}

La harina de zapallo presenta características nutricionales interesantes y es considerada un alimento funcional por su alto contenido de acarotenos, Bcarotenos, luteína, minerales, y ácidos grasos polinsaturados, el objetivo fue caracterizar la harina integral del zapallo para su posible uso en la alimentación de pollos de engorde y gallinas ponedoras ya que a partir de esto se podría influir en la pigmentación de la yema del huevo, piel y tarsos de pollo. En el análisis químico de la harina de zapallo se obtuvieron los siguientes resultados: humedad $13.24 \%$, proteína $4.63 \%$, grasas $1.82 \%$, ceniza $6.59 \%$, fibras $2.44 \%$, carbohidratos $71.28 \%$ y energía $320.02 \mathrm{kcal} / 100 \mathrm{mg}$, carotenoides totales $76,4 \mathrm{mg} / \mathrm{Kg}$. La harina integral de zapallo cumple con los estándares exigidos por la normativa nacional vigente para dicho producto, además presentó un alto contenido de nutrientes y pigmentos, ante lo cual puede ser incluido en la dieta de pollos de engorde y gallinas ponedoras.

Descriptores: Zapallo; Harina; Pigmentos; Alimento alternativo

\begin{abstract}
Pumpkin meal has interesting nutritional characteristics and is considered a functional food because of its high content of $\alpha$-carotenes, $\beta$-carotenes, lutein, minerals, and polyunsaturated fatty acids, the objective was to characterize the whole pumpkin flour for possible use in chicken feeding of fattening and laying hens since from this it could influence the pigmentation of the egg yolk, skin and chicken tarses. The following results were obtained in the chemical analysis of the pumpkin meal: moisture $13.24 \%$, protein $4.63 \%$, fats $1.82 \%$, ash $6.59 \%$, fibers $2.44 \%$, carbohydrates $71.28 \%$ and energy 320.02 $\mathrm{kcal} / 100 \mathrm{mg}$, total carotenoids $76.4 \mathrm{mg} / \mathrm{kg}$ Wholemeal pumpkin flour complies with the standards required by the current national regulations for this product, also presented a high content of nutrients and pigments, which can be included in the diet of broilers and laying hens.
\end{abstract}

Descriptors: Pumpkin; Flour; Pigments; Alternative food

\section{INTRODUCCIÓN}

La producción agropecuaria en el Ecuador es muy variada, siendo la ganadería y acuacultura sus mayores referentes; sin embargo, la actividad avícola ha sido uno de los sectores productivos con más desarrollo en el País; su consumo se encuentra en franco desarrollo en todo el territorio ecuatoriano, satisfaciendo las necesidades 


\section{CIENCIAMATRIA}

Revista Interdisciplinaria de Humanidades, Educación, Ciencia y Tecnología

Año VI. Vol. VI. N9. Julio - Diciembre 2019

Hecho el depósito de ley: pp201602FA4721

ISSN-L: 2542-3029; ISSN: 2610-802X

Universidad Nacional Experimental Francisco de Miranda (UNEFM). Santa Ana de Coro. Venezuela

Freddy Alain Mendoza Rivadeneira; Roy Leonardo Barre Zambrano; Plinio Abelardo Vargas Zambrano; Limbert Ivan Zambrano Pinoargote

proteicas de la población (Pérez, 2013). La crianza de pollo de engorde es una de las actividades pecuarias más difundidas en el país, condiciones que permiten crear temas de investigación y producción de este sector (Errecart, 2014).

El costo de alimentación para la crianza de pollos de engorde en el país, actualmente constituye un $75 \%$ del gasto total de crianza, es debido a esta situación la importancia de investigar nuevos alimentos alternativos que disminuyan los costos en comparación con el uso de insumos alimenticios convencionales (Azcona, 2013). Dentro de la búsqueda de alimentos alternativos surge la posibilidad de sustituir estos por materias primas de uso común y que compiten con la alimentación humana, bajar los costos de producción y lograr un mejor margen de utilidad dentro de la producción avícola (Quiroz, 2004).

En el Ecuador existe variedad y géneros de frutas y vegetales que contienen gran valor nutricional, al ser un país con gran impulso agrícola; sin embargo, su población desconoce el valor nutricional de sus productos agrícolas, sus potenciales nutricionales y sobretodo como utilizarlos en la alimentación animal, sin que los mismos pierdan sus nutrientes (Ramos, 2013). En la búsqueda de alimentos alternativos se encuentran insumos con características nutritivas que puedan cumplir con este propósito y además tengan menor costo que las materias primas tradicionales (Lozano, 2011). A nivel internacional, específicamente en Colombia se utilizado la harina integral de zapallo (cucúrbita moschata), como alimento alternativo y de bajo costo, Carvajal Tapia, Martínez Mamian \& Vivas Quila (2017), destacan que:

La inclusión de harina de zapallo en dietas para alimentación de pollos de engorde mayor al $7,5 \%$ y menor a $15 \%$, se considera como una materia prima no convencional que contribuye a la pigmentación de la piel, sin afectar parámetros productivos (consumo de alimento, ganancia de peso y conversión alimenticia) en la etapa final, Además, la inclusión de harina de zapallo en dietas para pollos de engorde puede convertirse en una alternativa para los agro ecosistemas de economía campesina generando un valor agregado en términos de pigmentación de la piel de los pollos ( $p$. 99). 


\section{CIENCIAMATRIA}

Revista Interdisciplinaria de Humanidades, Educación, Ciencia y Tecnología

Año VI. Vol. VI. N9. Julio - Diciembre 2019

Hecho el depósito de ley: pp201602FA4721

ISSN-L: 2542-3029; ISSN: 2610-802X

Universidad Nacional Experimental Francisco de Miranda (UNEFM). Santa Ana de Coro. Venezuela

Freddy Alain Mendoza Rivadeneira; Roy Leonardo Barre Zambrano; Plinio Abelardo Vargas Zambrano; Limbert Ivan Zambrano Pinoargote

Se puede percibir como la harina de zapallo, puede suplementar la alimentación de pollos, sin alterar la constitución física de los mismos, manteniendo el rendimiento en proporción a peso, color, tamaño, costo, lo cual permite deducir que puede ser empleada en otros escenarios medio ambientales. Así mismo, Camilo Ubaque, Viviana Orozco, Ortiz, Piedad Valdés \& Alirio Vallejo (2015), advierten que para el procesamiento de este tipo de alimento alternativos,

Se requiere de una alta inversión en mano de obra y energía para el desarrollo del proceso, a través de métodos artesanales, aspecto que demanda de mejoras en innovación, que logren su optimización y aumenten la competitividad de esta materia prima (p. 143).

Lo cual puede ser causal para que los pequeños productores o quienes no puedan invertir en tecnificar el proceso, prefieran seguir usando alimentos convencionales que en relación costo/tiempo, podrían seguir siendo atractivos para el productor en aras de hacer inversiones para el mantenimiento y sostenimiento de sus granjas o unidades de producción, siendo necesaria proseguir con la experimentación de la harina de zapallo con la finalidad de gestionar mayores pruebas que permitan con la base científica a impulsar la inversión para la creación de plantas procesadoras a gran escala, colocando la harina de zapallo no solo como una alternativa nutricional, sino de adquisición el mercado a costos que puedan ser competitivos con la agroindustria tradicional.

Siendo esto posible por cuanto en el Ecuador se da una importante producción de zapallo, la provincia de mayor producción es Manabí con $11662 \mathrm{Ha}$, con una producción considerable de aproximadamente 12.577 Tm (MAGAP, 2014) y ésta hortaliza sólo se utiliza en la crianza de cerdos y para la elaboración de sopas (Andreas, 2007).

El zapallo (Cucurbita moschata.) contiene un $91 \%$ de humedad; sin embargo, este valor puede cambiar según el tiempo de madurez, su principal mineral es el potasio (Ramírez y Villa, 2015). Las semillas y flores del zapallo son comestibles, y cada uno de estos componentes tiene aportes importantes. Además, contiene vitaminas, carotenos, y otros 


\section{CIENCIAMATRIA}

Revista Interdisciplinaria de Humanidades, Educación, Ciencia y Tecnología

Año VI. Vol. VI. N9. Julio - Diciembre 2019

Hecho el depósito de ley: pp201602FA4721

ISSN-L: 2542-3029; ISSN: 2610-802X

Universidad Nacional Experimental Francisco de Miranda (UNEFM). Santa Ana de Coro. Venezuela

Freddy Alain Mendoza Rivadeneira; Roy Leonardo Barre Zambrano; Plinio Abelardo Vargas Zambrano; Limbert Ivan Zambrano Pinoargote

componentes como el licopeno, vitamina c, ácido fólico y cumarinas los cuáles aportan un beneficio a la salud de los consumidores (Ramos, 2013).

El componente principal del zapallo es el agua, es rico por su contenido en grasas y proteínas pueden utilizarse como fuente de hidratos de carbono contiene vitaminas: $A$, B1, B2, B5, C y aminoácidos esenciales, también contiene sales minerales como: fósforo, potasio, calcio, sodio, silicio, magnesio, hierro y cloro, se puede consumir en forma indirecta como materia prima para la agroindustria. Es de fácil digestión y aporta pocas calorías, por ello se los encuentran incluidos en la mayoría de las dietas alimenticias (Flores, 2015).

El fruto de la especie C. moschata, por su aporte de carotenoides naturales como alfa, beta caroteno y luteína, sería una alternativa para poder lograr una buena pigmentación de los pollos de engorde, sin tener que recurrir a los pigmentantes artificiales, con lo cual se obtendría en forma natural un pollo bien pigmentado (Miglierina, 2013). Los pigmentos son compuestos (carotenoides o xantofilas) que por su actividad tienden a cambiar la coloración de la piel de los pollos, la grasa subcutánea y la yema de huevo (Cuevas et. al., 2003).

El zapallo puede constituirse en una alternativa viable en comparación con harinas procesadas del maíz, el cual exige mayor inversión para su producción, aunado que el zapallo posee valores nutricionales importantes para contribuir en una dieta rica en vitaminas y minerales, tan necesario para el crecimiento óptimo de las crias para su posterior beneficio.

\section{METODOLOGÍA}

La presente investigación se realizó en la Universidad Técnica de Manabí, Facultad de Ciencias Zootécnicas, ubicada en la ciudad de Chone, provincia de Manabí, aplicándose un tipo de investigación aplicativa y un diseño experimental que permitió procesar una muestra de harina de zapallo como prueba piloto para el establecimiento de su caracterización, Hernández, Fernández y Batista (2014). Por lo que se procedió a 


\section{CIENCIAMATRIA}

Revista Interdisciplinaria de Humanidades, Educación, Ciencia y Tecnología

Año VI. Vol. VI. Nº. Julio - Diciembre 2019

Hecho el depósito de ley: pp201602FA4721

ISSN-L: 2542-3029; ISSN: 2610-802X

Universidad Nacional Experimental Francisco de Miranda (UNEFM). Santa Ana de Coro. Venezuela

Freddy Alain Mendoza Rivadeneira; Roy Leonardo Barre Zambrano; Plinio Abelardo Vargas Zambrano; Limbert Ivan Zambrano Pinoargote

generar un flujograma de proceso, el cual permitió concebir los pasos a desarrollar desde la recepción hasta la obtención de la muestra final. El proceso de elaboración de harina de zapallo comprendió las etapas que se muestran en la figura 1.

745 g zapallo

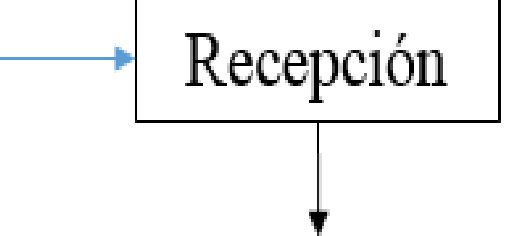

\section{Limpieza reducción de tamaño}

745 g zapallo con humedad $95.30 \%$

$37 \mathrm{~g}$ zapallo seco

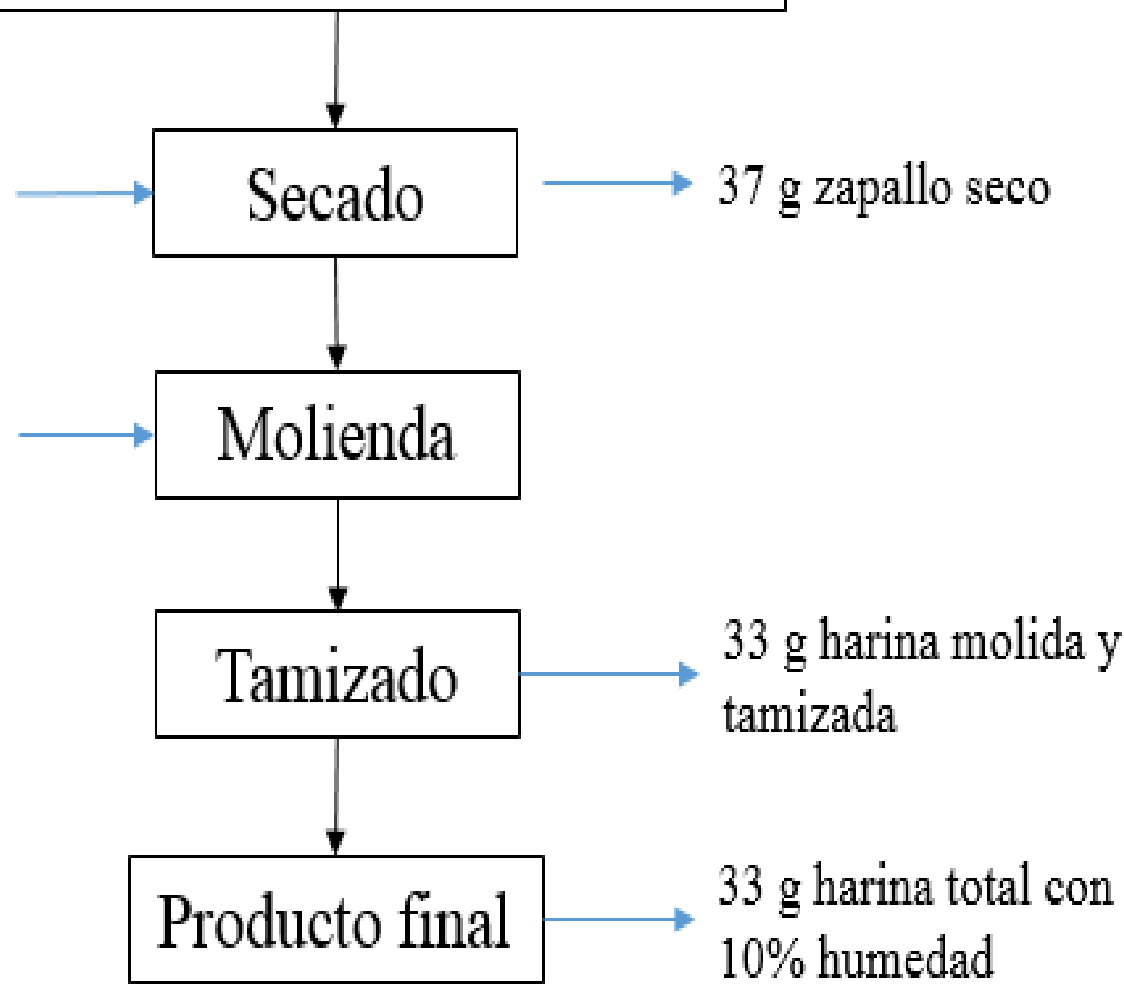

Figura 1. Flujograma proceso de obtención de harina

La harina se obtuvo mediante un proceso de deshidratación en un secador con circulación de aire, se molió y se tamizó a un tamaño de partícula de $0.5 \mathrm{~mm}$ según lo 


\section{CIENCIAMATRIA}

Revista Interdisciplinaria de Humanidades, Educación, Ciencia y Tecnología

Año VI. Vol. VI. N9. Julio - Diciembre 2019

Hecho el depósito de ley: pp201602FA4721

ISSN-L: 2542-3029; ISSN: 2610-802X

Universidad Nacional Experimental Francisco de Miranda (UNEFM). Santa Ana de Coro. Venezuela

Freddy Alain Mendoza Rivadeneira; Roy Leonardo Barre Zambrano; Plinio Abelardo Vargas Zambrano; Limbert Ivan Zambrano Pinoargote

establecido en la normativa INEN para harinas. En el caso, del proceso de secado de la materia prima para obtener harina de zapallo, se elaboraron rodajas grandes de $3 \mathrm{~mm}$ de grosor utilizando una rebanadora modelo IRAM LR 38324, HIG VOLTAGE, 110/220 V, luego utilizando un deshidratador eléctrico, elaborado por IMEGAR, INDUSTRIAL EXHAUST FAN modelo IEF-14, tamaño: 35 cm (14"), 110V $60 \mathrm{~Hz} 44 \mathrm{~W}$ capacidad de 10 bandejas se hizo la deshidratación del zapallo y de ahí pasó a la fase de molienda la cual se realizó en un molino eléctrico INDUSTRIAL EXHAUST FAN, modelo W112M $2201240 \mathrm{~V}$ con una capacidad de $40 \mathrm{~kg}$, se utilizaron los instrumentos de laboratorio como la balanza analítica digital modelo MSA1202S-100-D0 marca SARTORIUS.

Los ensayos se realizaron por triplicado en 3 días diferentes. Los análisis bromatológicos se realizaron mediante los protocolos INEN y AOAC, la cuantificación de los carotenoides totales se realizó por métodos espectofométricos.

Para la determinación de carotenoides totales se utilizó la metodología descrita por Synnøve y Jensen (1971), donde se pesó $1 \mathrm{~g}$ de la harina tamizada $(0,5 \mathrm{~mm})$ en un vaso de precipitado de $10 \mathrm{ml}$, se adicionan $25 \mathrm{ml}$ de etanol puro para análisis y se somete a extracción asistida por ultrasonidos durante 10 minutos. Se filtró cuantitativamente a través de un papel de filtro, lavando con pequeñas porciones de etanol y se coloca en embudo separador con $25 \mathrm{ml}$ de éter de petróleo (fracción volátil de 40-60). Se realizó la extracción: líquido - líquido y se separa la fase etérea. Se repite dicha extracción con $25 \mathrm{ml}$ más del éter de petróleo. Se unen las fases etéreas (volumen final $50 \mathrm{~mL}$ ). Se lee la absorbancia a $450 \mathrm{~nm}$ contra blanco de éter de petróleo.

Se calcula el contenido de carotenoides totales a través de la siguiente expresión:

$C=A \cdot V \cdot f \cdot 10 / 2500$

Donde:

$\mathrm{C}=$ cantidad de carotenoides en $\mathrm{mg}$

$A=$ absorbancia (en este caso fue de 0,382 como promedio de tres réplicas)

$\mathrm{V}=$ volumen final en mililitros (en este caso fue de $50 \mathrm{~mL}$ ) 


\section{CIENCIAMATRIA}

Revista Interdisciplinaria de Humanidades, Educación, Ciencia y Tecnología

Año VI. Vol. VI. N9. Julio - Diciembre 2019

Hecho el depósito de ley: pp201602FA4721

ISSN-L: 2542-3029; ISSN: 2610-802X

Universidad Nacional Experimental Francisco de Miranda (UNEFM). Santa Ana de Coro. Venezuela

Freddy Alain Mendoza Rivadeneira; Roy Leonardo Barre Zambrano; Plinio Abelardo Vargas Zambrano; Limbert Ivan Zambrano Pinoargote

$f=$ factor de dilución (en este caso fue 1)

10 = factor para la transformación de unidades

2500 = coeficiente de extinción promedio para los carotenoides bajo las condiciones de lectura de absorbancia.

\section{RESULTADOS}

Las propiedades fisicoquímicas de harina de zapallo ( $\mathrm{HZ}$ ) como humedad, proteína, grasa, ceniza, fibra, carbohidratos totales y energía fueron determinadas por un laboratorio de ensayo acreditado por el SAE ubicado en la ciudad de Quito, la descripción de la muestra fue un polvo color amarillento.

\section{Cuadro 1}

Características bromatológicas de la harina de zapallo

\begin{tabular}{lll} 
PARÁMETRO & MÉTODO & RESULTADO \\
\hline Humedad (\%) & PEE/LA/02 INEN ISO 712 & 13.24 \\
Proteína (\%) & PEE/LA/01 INEN ISO 20483 & 4.63 \\
Grasa (\%) & PEE/LA/05 INEN ISO 11085 & $1.82 \pm 0.03$ \\
Ceniza (\%) & PEE/LA/03 INEN 520 & 6.59 \\
Fibra (\%) & INEN 522 & 2.44 \\
$\begin{array}{l}\text { Carbohidratos totales } \\
\text { (\%) }\end{array}$ & Cálculo & 71.28 \\
Energía (kcal/100mg) & Cálculo & \\
\hline
\end{tabular}

En la determinación de carotenoides totales el cálculo realizado fue:

$C=A \cdot V \cdot f \cdot 10 / 2500$

$\mathrm{C}=0,382 \cdot 50 \cdot 1 \cdot 10 / 2500$

$\mathrm{C}=0,0764 \mathrm{mg}$ 


\section{CIENCIAMATRIA}

Revista Interdisciplinaria de Humanidades, Educación, Ciencia y Tecnología

Año VI. Vol. VI. N9. Julio - Diciembre 2019

Hecho el depósito de ley: pp201602FA4721

ISSN-L: 2542-3029; ISSN: 2610-802X

Universidad Nacional Experimental Francisco de Miranda (UNEFM). Santa Ana de Coro. Venezuela

Freddy Alain Mendoza Rivadeneira; Roy Leonardo Barre Zambrano; Plinio Abelardo Vargas Zambrano; Limbert Ivan Zambrano Pinoargote

El valor en miligramos está expresado por cada gramo de harina de zapallo; por lo tanto, al transformar las unidades a $\mathrm{mg} / \mathrm{kg}$, el resultado final es $76,4 \mathrm{mg}$ de carotenoides por cada kg de harina.

Según la Figura 1 al proceso de elaboración de harina ingresaron $17.2 \mathrm{Kg}$ de zapallo con una pérdida de $15,93 \mathrm{Kg}$; esto indica que el rendimiento es de $7,45 \%$, obteniendo $1,28 \mathrm{Kg}$ de harina de zapallo.

\section{CONCLUSIÓN}

La harina integral de zapallo tiene un excelente contenido nutricional y puede ser usado como alimento alternativo para sustituir parcialmente materias primas convencionales en la alimentación avícola. Además, por su contenido de carotenoides totales puede contribuir en la pigmentación de la piel y tarsos del pollo de engorde.

Se constituye en una alternativa viable para la cría de pollos, sin embargo más allá de los beneficios nutricionales que posee, es necesario indicar el alto costo de inversión que se tiene para producirlo a gran escala, lo cual impedirá su masificación, aceptación, en el mercado en el corto y mediano plazo, siendo necesario recurrir a planes de inversión, políticas públicas, diseñadas en el interés de promover diversos rubros alimenticios en razón de procesar reales alternativas de alimento al mercado nacional, por consiguiente, se debe proseguir con la experimentación de harina de zapallo, con la finalidad de publicitar sus aportes en el campo del Ecuador, siendo posible realizar ensayos con los productos para que conozcan de primera línea, los aportes del zapallo.

\section{REFERENCIAS CONSULTADAS}

1. Andreas, W. (2007). Asegurando nuestro futuro. Litografía e Imprenta LIL S.A.

2. Azcona, Á. (2013). Manual de Nutrición y Dietética. Departamento de NutriciónM-008157. Madrid. 


\section{CIENCIAMATRIA}

Revista Interdisciplinaria de Humanidades, Educación, Ciencia y Tecnología

Año VI. Vol. VI. N9. Julio - Diciembre 2019

Hecho el depósito de ley: pp201602FA4721

ISSN-L: 2542-3029; ISSN: 2610-802X

Universidad Nacional Experimental Francisco de Miranda (UNEFM). Santa Ana de Coro. Venezuela

Freddy Alain Mendoza Rivadeneira; Roy Leonardo Barre Zambrano; Plinio Abelardo Vargas Zambrano; Limbert Ivan Zambrano Pinoargote

3. Carvajal Tapia, J., Martínez Mamian, C., \& Vivas Quila, N. J. (2017). Evaluación de parámetros productivos y pigmentación en pollos alimentados con harina zapallo (cucurbita moschata). Biotecnología En El Sector Agropecuario Y Agroindustrial, 15(2), 93-100. https://doi.org/10.18684/BSAA(15)93-100

4. Camilo Ubaque, C., Viviana Orozco, L., Ortiz, S., Piedad Valdés, M., \& Alirio Vallejo, F. (2015). SUSTITUCIÓN DEL MAÍZ POR HARINA INTEGRAL DE ZAPALLO EN LA NUTRICIÓN DE POLLOS DE ENGORDE. Revista U.D.C.A Actualidad \& Divulgación Científica, 18(1). Recuperado a partir de https://revistas.udca.edu.co/index.php/ruadc/article/view/462

5. Cuevas, B.; Díaz, G.; Molina, A. y Retamal, C. (2033). Pigmentos utilizados en raciones de gallinas ponedoras. Disponible en: https://www.biblioteca.org.ar/libros/8911.pdf

6. Errecart, V. (2014). Análisis del mercado mundial de carnes. CERE-Escuela de Economía y Negocios, 35-38.

7. Hernández, R., Fernández, C., Baptista, P. (2014). Metodología de la Investigación. McGraw Hill. Colombia.

8. Ramírez, E. y Villa, F. (2015). Obtención de Harina de zapallo por el proceso de secado de alimentos. Ventana Científica Vol. 5. N9 ISSN 2305-6010, 1-17.

9. Flores, J. (2015). Evaluación de la eficiencia de tres fertilizantes edáficos sobre el rendimiento y calidad del zapallo (Cucurbita maxima var. Unapal- Mandarino). Revista de Investigación Agraria y Ambiental, 185.

10. Lozano, J. (2011). La nutrición es Con-Ciencia. Universidad de Murcia. España.

11. MAGAP. (2014). Producción Nacional de Hortalizas. Ecuador.

12. Miglierina, A. (2013). Manual del cultivo de Zapallo anquito (Cucurbita moschata Duch). Argentina: INTA, E-BOOK. 7-25.

13. Pérez, M. (2013). Manual de prácticas de laboratorio Tecnología de Carne. Primera Edición. México.

14. Quiroz, E. (2004). Introducción a la Tecnología de Alimentos. Limusa. México. 83. 


\section{CIENCIAMATRIA}

Revista Interdisciplinaria de Humanidades, Educación, Ciencia y Tecnología

Año VI. Vol. VI. N9. Julio - Diciembre 2019

Hecho el depósito de ley: pp201602FA4721

ISSN-L: 2542-3029; ISSN: 2610-802X

Universidad Nacional Experimental Francisco de Miranda (UNEFM). Santa Ana de Coro. Venezuela

Freddy Alain Mendoza Rivadeneira; Roy Leonardo Barre Zambrano; Plinio Abelardo Vargas Zambrano; Limbert Ivan Zambrano Pinoargote

15. Ramos, E. (2013). Estudio del zapallo y aplicación a la repostería. Quito, Ecuador.

16. Synnøve L. and Jensen, A. (1971). Quantitative determination of carotenoids in photosynthetic tissues. Methods in Enzymology. Volume 23, Pages 586-602.

\section{REFERENCES CONSULTED}

1. Andreas, W. (2007). Securing our future Litografía e Imprenta LIL S.A.

2. Azcona, Á. (2013). Manual of Nutrition and Dietetics. Department of Nutrition-M008157. Madrid.

3. Carvajal Tapia, J., Martínez Mamian, C., \& Vivas Quila, N. J. (2017). Evaluation of productive parameters and pigmentation in chickens fed with pumpkin flour (cucurbita moschata). Biotechnology in the Agricultural and Agroindustrial Sector, 15 (2), 93-100. https://doi.org/10.18684/BSAA(15)93-100

4. Camilo Ubaque, C., Viviana Orozco, L., Ortiz, S., Piedad Valdés, M., \& Alirio Vallejo, F. (2015). REPLACEMENT OF CORN BY INTEGRAL SHOE FLOUR IN NUTRITION OF CHICKENS. U.D.C.A Magazine News \& Scientific Dissemination, 18 (1). 18 Recovered from https://revistas.udca.edu.co/index.php/ruadc/article/view/462

5. Caves, B .; Díaz, G .; Molina, A. and Retamal, C. (2033). Pigments used in rations of laying hens. Available at: https://www.biblioteca.org.ar/libros/8911.pdf

6. Errecart, V. (2014). Analysis of the world meat market. CERE-School of Economics and Business, 35-38.

7. Hernández, R., Fernández, C., Baptista, P. (2014). Investigation methodology. McGraw Hill Colombia.

8. Ramírez, E. and Villa, F. (2015). Obtaining pumpkin flour by the process of drying food. Scientific Window Vol. 5. N 9 ISSN 2305-6010, 1-17.

9. Flores, J. (2015). Evaluation of the efficiency of three edaphic fertilizers on the yield and quality of the pumpkin (Cucurbita maxima var. Unapal-Mandarino). Agricultural and Environmental Research Magazine, 185. 


\section{CIENCIAMATRIA}

Revista Interdisciplinaria de Humanidades, Educación, Ciencia y Tecnología

Año VI. Vol. VI. N9. Julio - Diciembre 2019

Hecho el depósito de ley: pp201602FA4721

ISSN-L: 2542-3029; ISSN: 2610-802X

Universidad Nacional Experimental Francisco de Miranda (UNEFM). Santa Ana de Coro. Venezuela

Freddy Alain Mendoza Rivadeneira; Roy Leonardo Barre Zambrano; Plinio Abelardo Vargas Zambrano; Limbert Ivan Zambrano Pinoargote

10. Lozano, J. (2011). Nutrition is Con-Science. University of Murcia. Spain.

11. MAGAP (2014). National Vegetable Production. Ecuador.

12. Miglierina, A. (2013). Manual of the cultivation of Zapallo anquito (Cucurbita moschata Duch). Argentina: INTA, E-BOOK. 7-25.

13. Pérez, M. (2013). Manual of practices of Meat Technology laboratory. First edition. Mexico.

14. Quiroz, E. (2004). Introduction to Food Technology. Limusa Mexico. 83.

15. Ramos, E. (2013). Study of the pumpkin and application to baking. Quito, Ecuador.

16. Synnøve L. and Jensen, A. (1971). Quantitative determination of carotenoids in photosynthetic tissues. Methods in Enzymology. Volume 23, Pages 586-602.

C2019 por los autores. Este artículo es de acceso abierto y distribuido según los términos y condiciones de la licencia Creative Commons Atribución-NoComercial-Compartirlgual 4.0 Internacional (CC BY-NC-SA 4.0)

(https://creativecommons.org/licenses/by-nc-sa/4.0/). 